\title{
The effective conductivity of arrays of squares: large random unit cells and extreme contrast ratios
}

\author{
Johan Helsing ${ }^{1}$ \\ Numerical Analysis, Centre for Mathematical Sciences, \\ Lund University, Box 118, SE-221 00 LUND, Sweden
}

\begin{abstract}
An integral equation based scheme is presented for the fast and accurate computation of effective conductivities of two-component checkerboard-like composites with complicated unit cells at very high contrast ratios. The scheme extends recent work on multi-component checkerboards at medium contrast ratios. General improvement include the simplification of a longrange preconditioner, the use of a banded solver, and a more efficient placement of quadrature points. This, together with a reduction in the number of unknowns, allows for a substantial increase in achievable accuracy as well as in tractable system size. Results, accurate to at least nine digits, are obtained for random checkerboards with over a million squares in the unit cell at contrast ratio $10^{6}$. Furthermore, the scheme is flexible enough to handle complex valued conductivities and, using a homotopy method, purely negative contrast ratios. Examples of the accurate computation of resonant spectra are given.
\end{abstract}

Keywords: Random checkerboard, Homogenization, Integral equation, Fast solver, Metamaterial

\section{Introduction}

This paper is devoted to solving the electrostatic equation for periodic composites with unit cells made of squares of conductivity $\sigma_{2}$ that are ei-

Email address: helsing@maths.1th.se (Johan Helsing)

URL: http://www.maths.1th.se/na/staff/helsing/ (Johan Helsing)

${ }^{1}$ Tel.:+46 462223372 
ther mixed with other squares of conductivity $\sigma_{1}$, to form a checkerboard structure, or simply embedded in a background material of conductivity $\sigma_{1}$. There are $N_{\mathrm{sq}}$ squares in the unit cell and the area fraction of squares with conductivity $\sigma_{2}$ is denoted $p$. The goal is to compute the effective conductivity $\sigma_{*}$ rapidly, with high accuracy, and for almost any combination of $\sigma_{1}$, $\sigma_{2}$, and $p$ for which the electrostatic equation has a solution.

\subsection{Motivation and challenges}

There are several applications that motivate our study. The homogenization of checkerboards with random unit cells at high real valued contrast ratios $\sigma_{2} / \sigma_{1}$ (or $\left.\sigma_{1} / \sigma_{2}\right)$ is a classic problem in materials science. It is of interest to study how $\sigma_{*}$ depends on $p$ and in particular what happens when one type of squares forms a connected path throughout the composite (percolation). The contrast ratio can be considerable in materials of technological importance. A ratio of $10^{7}$ is not unusual [23]. Very large $N_{\text {sq }}$ are then needed to reach convergence to statistical limits. See Chapters 10.10 and 10.11 of [25] for a review of this field. In the metamaterial community there is a strong interest in a related issue, namely how to compute resonant spectra of effective dielectric permittivity functions (spectra of plasmonic excitations) for composites made of polygonal metamaterial inclusions embedded in a dielectric background material [7, 27]. The electrostatic equation is the same for conducting and for dielectric materials. Only the notation differs, see Table on p. 19 of [25]. For simplicity, we will talk about conductivity in this context, too. Of particular interest is the behavior of $\sigma_{*}$ close to values of $\sigma_{2} / \sigma_{1}$ where the electrostatic equation does not have a solution or only has a solution as a limit in the complex $\sigma_{2} / \sigma_{1}$-plane.

The computational tasks just discussed offer extreme challenges. Nonsmooth interfaces tend to make solutions singular and hard to resolve. The electric fields close to certain corner vertices may just barely be square integrable. As $N_{\text {sq }}$ grows, the interaction between distantly separated parts in the computational domain may cause problems which cannot be resolved by discretization and local techniques alone. Being in the vicinity of parameter combinations where the electrostatic equation ceases to have a solution is often hard. All these difficulties add up and may manifest themselves as artificial ill-conditioning, slow convergence with mesh refinement, critical slowing down in iterative solvers, and severe loss of precision. Several methods have been suggested to alleviate these problems including variants of the finite element method [1, 6], network models [23, 12], renormalization 
schemes [20], mode-matching methods [27], and Brownian motion simulation [21]. See also Section 3 of [26] for state-of-the-art algorithms to combat critical slowing down in network models and [8] for a discussion of future directions in the research field at large.

\subsection{Our scheme}

Let $\Gamma$ denote the boundary (the interfaces) of a composite. We shall reformulate the electrostatic equation as a Fredholm second kind integral equation

$$
(I+K) \mu(z)=g(z), \quad z \in \Gamma,
$$

where $I$ is the identity, $K$ is an integral operator which is compact on smooth $\Gamma, \mu(z)$ is an unknown layer density, and $g(z)$ is a right hand side.

Solvers for large-scale boundary values problems on smooth domains often rely on integral equation reformulations of the form (1). The last few years have seen increased activity in the development of efficient solvers using (1) also when $\Gamma$ is non-smooth. The scheme of the present paper originates from work on non-smooth inclusion problems in free-space [17]. The ideas in [17] were later improved and extended to encompass the biharmonic equation [18], mixed boundary conditions [13], singular integral equations with non-zero indices [14], and boundaries with quadruple-junctions [15]. The present paper is a direct sequel to [15]. As in [15], we apply a combination of short- and long-range preconditioners to (1). Major new features include:

- A better strategy for choosing quadrature nodes which makes the error in $\sigma_{*}$ grow linearly with contrast ratio. In [15] the growth is superlinear.

- An improved long-range preconditioner which makes the computational cost grow almost linearly with $N_{\text {sq }}$. In [15] the growth is cubic.

- A homotopy-type method which allows for computing $\sigma_{*}$ at points in the complex $\sigma_{2} / \sigma_{1}$-plane where the solution to the electrostatic equation only exists as a non-unique limit.

In addition there are several minor improvements.

\subsection{Relation to the Bremer-Rokhlin scheme}

Other recent work on the efficient solution of (11) in the presence of non-smooth boundaries includes [5], which exploits cancellation of singularities, and a comprehensive mechanism currently being developed by a group 
around Bremer and Rokhlin [2, 3, 4]. Let us discuss the relation of the Bremer-Rokhlin scheme to our scheme.

Both schemes take as a starting point the observation that an accurate and economical discretization of (1) can only be effected by restricting the operator on the left hand side to a finite-dimensional subspace, determined by the right hand side $g(z)$. High resolution in combination with compression is used as a means to achieve this. The result is a kind of precomputed purpose-made composite quadrature, suitable for Nyström discretization.

The Bremer-Rokhlin scheme employs an elaborate machinery to construct families of 'universal quadratures'. Each universal quadrature is appropriate for the discretization of a given integral equation over an entire class of boundary segments with complicated geometry. When the integral equation depends on material parameters, in addition to geometry, more universal quadratures are needed. The approach has the advantage that the precomputation is done once and for all and can be stored on disk. When solving a particular problem involving many boundary singularities of similar shapes, only a few universal quadratures need to be activated. Our scheme precomputes 'quadrature-weighted inverses' afresh around every boundary singularity. This offers greater flexibility when applying the scheme to new situations and opens up for a parallel implementation, but requires more RAM storage.

Another difference between the two schemes is the way in which the process of resolution and compression is carried out. The Bremer-Rokhlin compression is done via a series of solutions of large linear systems followed by rank-revealing $Q R$ decompositions. Our scheme deals with resolution and compression in tandem, using a fast and stable recursion. No large linear systems are ever set up. This is an advantage for boundary segments where extremely high resolution is needed.

The schemes also differ in the assumptions made on $g(z)$. The BremerRokhlin scheme assumes that $g(z)$ is a restriction to $\Gamma$ of a function that satisfies the underlying partial differential equation in a neighborhood of each point on $\Gamma$. This assumption applies, for example, to certain important acoustic scattering problems. Our scheme only assumes that $g(z)$ is piecewise smooth. This is an advantage when the computational domain models granular materials or materials containing branching cracks.

An open question is how easily the two schemes generalize to three dimensions. Perhaps one can combine their best features? 


\subsection{Organization of the paper}

The paper is divided into eight sections. Section 2 introduces unit cells and integral equations that will be used in all examples. Section 3 is on discretization. The leading ideas in our compression scheme are summarized in Sections 4 and 5. Section 6 is on implementation. This material is essential for the understanding of how limits are taken in the complex $\sigma_{2} / \sigma_{1^{-}}$ plane and how the compression of inverses of giant matrices corresponding to intensely resolved integral operators can be executed in sub-linear time. Section 7 presents improvements to the long-range preconditioner proposed in [15]. The paper ends in Section 8 with some truly large-scale and accurate numerical examples for random checkerboards along with the computation of resonant spectra of two metamaterial composites. The reader interested in more examples is referred to a forthcoming paper [16].

\section{Integral equations for the electrostatic problem}

We shall solve the electrostatic partial differential equation on three types of doubly-periodic domains in a plane $D$ : square arrays of squares, staggered arrays of squares, and two-component random checkerboards. An average electric field $e=\left(e_{x}, e_{y}\right)$ of unit strength is applied to $D$ and we seek the potential $U(r)$ for the computation of $\sigma_{*}$ in direction $e$

$$
\sigma_{*}=\int_{D_{0}}(\sigma(r) \nabla U(r) \cdot e) \mathrm{d} V_{r},
$$

where $\sigma(r)$ is the local conductivity, $\mathrm{d} V_{r}$ is an infinitesimal area element, and the unit cell $D_{0}$ is $[-1 / 2,1 / 2) \times[-1 / 2,1 / 2)$. We make no distinction between points or vectors in a real plane $\mathbb{R}^{2}$ and points in a complex plane $\mathbb{C}$. From now on, all points will be denoted $z$ or $\tau$.

The interfaces $\Gamma$ in $D$ are given orientation. The restriction of $\Gamma$ to $D_{0}$ is denoted $\Gamma_{0}$ and the outward unit normal of $\Gamma$ at $z$ is $n_{z}=n(z)$. Corner vertices are denoted $\gamma_{k}$. Obviously, $\sigma(z)$ may jump as $\Gamma$ is crossed. Let $\sigma_{+}(z)$ denote the conductivity on the positive side of $\Gamma$ at $z$, let $\sigma_{-}(z)$ denote the conductivity on the negative side, and introduce as in [15]

$$
\begin{aligned}
& a(z)=\sigma_{+}(z)-\sigma_{-}(z), \quad z \in \Gamma, \\
& b(z)=\sigma_{+}(z)+\sigma_{-}(z), \quad z \in \Gamma, \\
& c(z)=\sigma_{+}(z) \sigma_{-}(z), \quad z \in \Gamma, \\
& \lambda(z)=a(z) / b(z), \quad z \in \Gamma .
\end{aligned}
$$



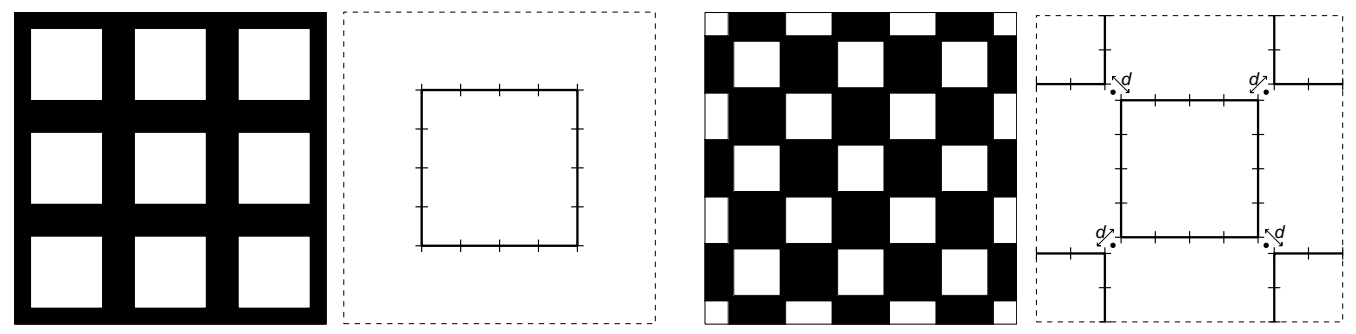

Figure 1: Left: a cutout of a square array of squares with $p=0.5$ and a unit cell with a 16-panel coarse mesh on $\Gamma_{0}$. Right: a staggered array of squares with $p=0.4$ and a unit cell with a 32-panel coarse mesh on $\Gamma_{0}$. The vertex separation distance is $d$ and the dots indicate double-corner concentration points $\delta_{k}$.

Our domains exhibit similarities, but they also differ in important respects. Different integral equation reformulations will be used for efficiency.

\subsection{Ordered arrays of squares}

The two ordered arrays are made by placing squares with conductivity $\sigma_{2}$ in a plane with conductivity $\sigma_{1}$. Fig. 1 shows cutouts of unit cells. The orientation of $\Gamma$ is positive. The arrays are overall isotropic, so $\sigma_{*}$ is independent of $e$. The points in between neighboring corner vertices in the staggered array are called double-corner concentration points and denoted $\delta_{k}$.

The conductivity $\sigma_{2}$ may be complex valued while $\sigma_{1}$ is assumed real. The special case of real valued and negative ratios $\sigma_{2} / \sigma_{1}$ poses a particular challenge. The electrostatic equation may not have a unique solution and this property is then carried over to the integral equation. Sometimes $\sigma_{*}$, viewed as a function of $\sigma_{2}$ with $\sigma_{1}$ held constant, has a well defined limit which depends on whether $\sigma_{2} / \sigma_{1}$ approaches the negative real axis from above or from below in the complex plane. Hetherington and Thorpe [19] argue that such a branch cut occurs for $\sigma_{*}$ of composites with right-angled interfaces whenever $\sigma_{2} / \sigma_{1} \in[-3,-1 / 3]$. See also p. 378 of Milton [25]. We shall capture the limit of $\sigma_{*}$ from above.

We follow standard practice for inclusion problems and represent $U(z)$ as a continuous function which is a sum of a driving term and a single-layer potential with density $\rho(z)[10]$. Enforcing continuity of the normal current across $\Gamma$ we arrive at the integral equation

$$
\rho(z)+\frac{\lambda(z)}{\pi} \int_{\Gamma} \rho(\tau) \Im\left\{\frac{n_{z} \bar{n}_{\tau} \mathrm{d} \tau}{\tau-z}\right\}=2 \lambda(z) \Re\left\{\bar{e} n_{z}\right\}, \quad z \in \Gamma_{0},
$$



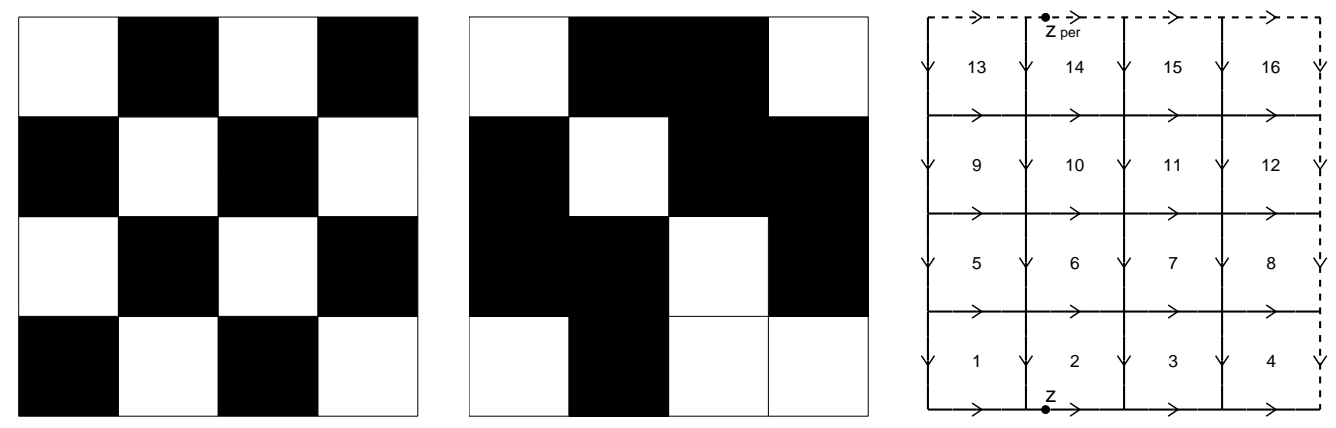

Figure 2: Left: a cutout of a two-component ordered checkerboard. Middle and right: A unit cell $D_{0}$ of a random checkerboard with $N_{\mathrm{sq}}=16$ squares. Orientation of $\Gamma_{0}$ (solid lines) and $L_{0} \backslash \Gamma_{1}$ (dashed lines) along with a point $z \in \Gamma_{1}$ and its periodic image $z_{\text {per }} \in L_{0} \backslash \Gamma_{1}$.

where the 'bar' symbol denotes complex conjugation. We observe that (7) is a Fredholm integral equation of the second kind with an integral operator which is compact away from the corner vertices.

The parameter $\lambda(z)$ in (7) is independent of $z$. Should the integral operator in (7) have been compact everywhere, then, in a finite portion of the complex $\lambda$-plane, there could exist a finite number of values $|\lambda| \geq 1$, called eigenvalues of the equation, for which the solution $\rho(z)$ may not be unique or may not even exist as a limit. See Sections 8 and 38 of Mikhlin [24]. If, however, (77) can be solved for $\rho(z)$ and under the assumption that the inclusions do not overlap the unit cell boundary, the effective conductivity can be computed from

$$
\sigma_{*}=\sigma_{1}+\sigma_{1} \int_{\Gamma_{0}} \rho(z) \Re\{\bar{e} z\} \mathrm{d}|z| .
$$

Depending on how the unit cell is chosen, the squares in the staggered array may overlap the unit cell boundary. With the choice in Fig. 1, they certainly do. But since the layer density $\rho(z)$ is periodic and identical on all squares one can modify (8) so that it integrates $\rho(z)$ twice on the square at the center of the unit cell and ignores $\rho(z)$ on the other squares.

\subsection{Checkerboards}

Fig. 2 shows checkerboards. The squares in $D$ have either high conductivity $\sigma_{2}$ or low conductivity $\sigma_{1}$. Here $\sigma_{2}$ and $\sigma_{1}$ are real so that $\sigma_{2} / \sigma_{1}>1$. 

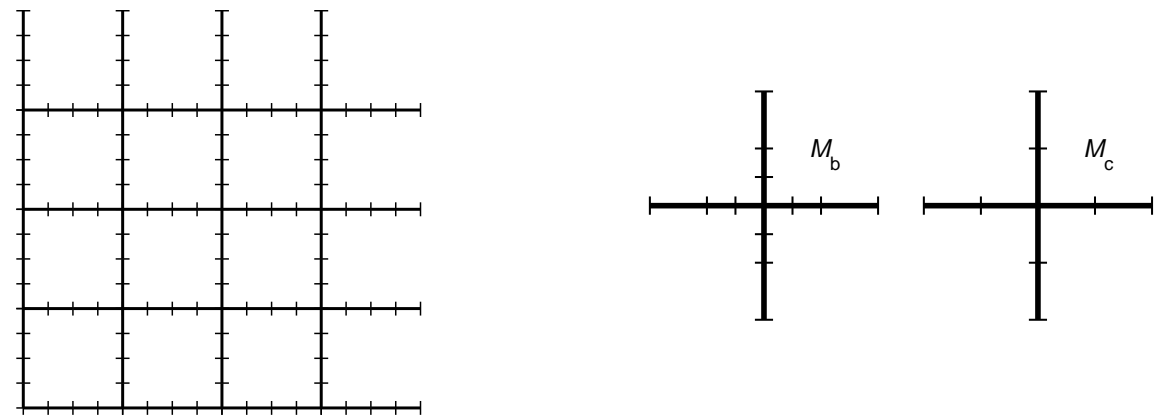

Figure 3: Left: a coarse mesh on $\Gamma_{0}$ for a checkerboard unit cell with $N_{\mathrm{sq}}=16$. There are four quadrature panels on each square side. Right: local meshes $\mathcal{M}_{\mathrm{b}}$ and $\mathcal{M}_{\mathrm{c}}$ centered around a corner vertex. There will be 192 discretization points on $\mathcal{M}_{\mathrm{b}}$ and 128 points on $\mathcal{M}_{\mathrm{c}}$.

The challenge is to achieve linear complexity and high accuracy in difficult situations.

The middle image of Fig. 2 is from a random checkerboard with $N_{\mathrm{sq}}=16$. The right image indicates $\Gamma_{0}$ by solid lines. The boundary of $D_{0}$ is denoted $L_{0}$ and $\Gamma_{1}=\Gamma_{0} \cap L_{0}$. Note that some or all squares that meet at a corner vertex $\gamma_{k}$ could have the same conductivity. Vertices where two squares of conductivity $\sigma_{1}$ and two squares of conductivity $\sigma_{2}$ meet diagonally, like in the left image of Fig. 2, will be referred to as special corner vertices.

An efficient Fredholm second kind integral equation for checkerboard problems can be derived by applying Green's third identity to the periodic function $U(z)-\Re\{\bar{e} z\}$. In terms of a transformed potential $u(z)$, this doublelayer type equation assumes the simple form

$$
u(z)-\frac{\lambda(z)}{\pi} \int_{\Gamma} u(\tau) \Im\left\{\frac{\mathrm{d} \tau}{\tau-z}\right\}=2 \frac{c(z)}{b(z)} \Re\left\{\bar{e} l_{0}(z)\right\}, \quad z \in \Gamma_{0},
$$

where $l_{0}(z)$ is zero for $z \in \Gamma_{0} \backslash \Gamma_{1}$ and equal to the vector difference of $z$ and its periodic image $z_{\text {per }} \in L_{0} \backslash \Gamma_{1}$ for $z \in \Gamma_{1}$, see Section 2.2 of [15]. The effective conductivity can be computed from

$$
\sigma_{*}=\int_{\Gamma_{0}} u(z) \Im\{\bar{e} \mathrm{~d} z\}
$$

We observe that the integral operator in (9) is compact away from the corner vertices. 


\section{Discretization}

We discretize (77) and (9) using a Nyström scheme based on composite polynomial interpolatory quadrature and a parameterization $z(t)$ of $\Gamma_{0}$. Coarse meshes with four quadrature panels per square side are constructed on $\Gamma_{0}$, see Figs. 1 and 3. We also need fine meshes obtained from coarse meshes by subdividing panels neighboring corner vertices $n_{\text {sub }}$ times in a direction towards the vertices.

The layer densities $\rho(z)$ and $u(z)$ in (7) and (9) are smooth on most quadrature panels. We choose quadrature nodes and weights according to composite 16-point Gauss-Legendre quadrature in parameter $t$ on such panels. This quadrature has panelwise polynomial degree 31.

Panels neighboring corner vertices of ordered arrays of squares or special corner vertices of checkerboards require special attention and will be referred to as special panels. The layer densities $\rho(z)$ and $u(z)$ may undergo rapid changes there. This is so because of strong singularities that arise in $\nabla U(z)$. For checkerboards, as $\sigma_{2} / \sigma_{1} \rightarrow \infty$, this field is barely square integrable in $D_{0}$ and barely absolutely integrable on $\Gamma_{0}$, see Section 2.3 of [15]. See p. 378 of Milton [25] for a discussion of similar singularities that arise at corner vertices as $\sigma_{2} / \sigma_{1}$ approaches values in the range $[-3,-1 / 3]$.

Legendre nodes are not optimal for capturing the behavior of layer densities on special panels. Rather, it pays off to bunch quadrature nodes in a direction towards the vertices. An experimental investigation, see Section 8.2 , shows that nodes corresponding to zeros of the Jacobi polynomial $P(x)_{16}^{(\alpha, \beta)}$ on the canonical interval $x \in[-1,1]$, for certain $\alpha$ and $\beta$, are more efficient. For checkerboards and with the corner vertex at a special panel's right endpoint, we take $\alpha=\left(\sigma_{1} / \sigma_{2}\right)^{0.4}-1$ and $\beta=0$. With the corner vertex at a special panel's left endpoint, we take $\alpha=0$ and $\beta=\left(\sigma_{1} / \sigma_{2}\right)^{0.4}-1$. For ordered arrays of squares at negative contrast ratios we take $\alpha=10^{-6}-1$ and $\beta=0$ or $\alpha=0$ and $\beta=10^{-6}-1$. The corresponding quadrature weights are determined so that the panelwise polynomial degree is 15 .

A discretization in parameter $t$ on the coarse mesh of a checkerboard gives $N_{\text {coa }}=128 N_{\text {sq }}$ points $z_{i}=z\left(t_{i}\right)$ and the same number of weights $w_{i}$. On the fine mesh there are $N_{\text {fin }}=\left(128+64 n_{\text {sub }}\right) N_{\text {sq }}$ discretization points. The square array of squares has $N_{\text {coa }}=256$ and $N_{\text {fin }}=256+64 n_{\text {sub }}$. The staggered array of squares has $N_{\text {coa }}=512$ and $N_{\text {fin }}=512+128 n_{\text {sub }}$. We collect quadrature weights on the diagonal of matrices $\mathbf{W}$ for later use. The subscripts 'coa' and 'fin' are used to indicate the coarse mesh and the refined mesh, respectively. 


\section{Short-range preconditioning}

Consider now the Fredholm second kind integral equations (7) and (9) in the general form (11) where $g(z)$ is piecewise smooth. Let $K(\tau, z)$ denote the kernel of $K$. Split $K(\tau, z)$ into two functions

$$
K(\tau, z)=K^{\star}(\tau, z)+K^{\circ}(\tau, z)
$$

where $K^{\star}(\tau, z)$ is zero except for when $\tau$ and $z$ simultaneously lie in a neighborhood $\Gamma_{k}^{\star}$ centered around a particular $\gamma_{k}$ or $\delta_{k}$. Then $K^{\circ}(\tau, z)$ is zero. The neighborhoods $\Gamma_{k}^{\star}$ cover four coarse panels around $\gamma_{k}$ of a square array of squares and eight coarse panels around $\delta_{k}$ of a staggered array of squares and around $\gamma_{k}$ of a checkerboard. Compare Section 3.2 of [15].

The kernel split (11) corresponds to an operator split $K=K^{\star}+K^{\circ}$ where $K^{\circ}$ is a compact operator. After discretization (1) assumes the form

$$
\left(\mathbf{I}+\mathbf{K}^{\star}+\mathbf{K}^{\circ}\right) \boldsymbol{\mu}=\mathbf{g},
$$

where $\mathbf{I}, \mathbf{K}^{\star}$, and $\mathbf{K}^{\circ}$ are square matrices and $\boldsymbol{\mu}$ and $\mathbf{g}$ are columns vectors. Note that $\mathbf{K}^{\star}$ is sparse and block diagonal. The blocks of $\mathbf{K}_{\text {coa }}^{\star}$ corresponding to $\gamma_{k}$ of a square array of squares have size $64 \times 64$ while the blocks corresponding to $\delta_{k}$ of a staggered array of squares and to $\gamma_{k}$ of a checkerboard have size $128 \times 128$.

The change of variables

$$
\mu(z)=\left(I+K^{\star}\right)^{-1} \tilde{\mu}(z)
$$

makes (12) read

$$
\left(\mathbf{I}+\mathbf{K}^{\circ}\left(\mathbf{I}+\mathbf{K}^{\star}\right)^{-1}\right) \tilde{\boldsymbol{\mu}}=\mathbf{g} .
$$

This right preconditioned equation corresponds to the discretization of a Fredholm second kind equation with a composed compact operator and the solution $\tilde{\boldsymbol{\mu}}$ is the discretization of a piecewise smooth function. There should be no ill-conditioning in (14) due to mesh refinement close to corner vertices and we can view $\left(\mathbf{I}+\mathbf{K}^{\star}\right)^{-1}$ as a short-range preconditioner for (12). There will, however, be ill-conditioning in (14) for parameter values $\lambda(z)$ that are very close to eigenvalues of (7) and (91). 


\section{Compression of the preconditioned equation}

The matrix $\mathbf{K}^{\circ}$ and the right hand side $\mathbf{g}$ in (14) can be accurately evaluated on a grid on the coarse mesh. Only $\left(\mathbf{I}+\mathbf{K}^{\star}\right)^{-1}$ needs a grid on the refined mesh for its accurate evaluation. We introduce the compressed weighted inverse

$$
\mathbf{R}=\mathbf{P}_{W}^{T}\left(\mathbf{I}_{\text {fin }}+\mathbf{K}_{\text {fin }}^{\star}\right)^{-1} \mathbf{P} .
$$

Here $\mathbf{P}$ is a prolongation operator from the coarse grid to the fine grid, $\mathbf{P}_{W}=\mathbf{W}_{\text {fin }} \mathbf{P} \mathbf{W}_{\text {coa }}^{-1}$ is a weighted prolongation operator, see Section 5 of [13]. Furthermore, the block-diagonal $N_{\text {coa }} \times N_{\text {coa }}$ matrix $\mathbf{P}_{W}^{T} \mathbf{P}$, where superscript $T$ denotes the transpose, has the property

$$
\mathbf{P}_{W}^{T} \mathbf{P}=\mathbf{I}
$$

Strictly speaking, the relation (16) does not hold exactly for matrix blocks corresponding to special panels. It holds, however, also for these blocks that

$$
\mathbf{f}_{i} \mathbf{W}_{\text {соа }} \mathbf{P}_{W}^{T} \mathbf{P} \mathbf{f}_{j}=\mathbf{f}_{i} \mathbf{W}_{\text {соа }} \mathbf{f}_{j},
$$

where $\mathbf{f}_{i}$ and $\mathbf{f}_{j}$ are discretizations of piecewise polynomials on the coarse grid of degree $i$ and $j$ and $i+j \leq 15$. One can say that (16) holds to the same polynomial degree as the overall quadrature holds.

With (15), equation (14) assumes the form

$$
\left(\mathbf{I}_{\text {coa }}+\mathbf{K}_{\text {coa }}^{\circ} \mathbf{R}\right) \tilde{\boldsymbol{\mu}}_{\text {coa }}=\mathbf{g}_{\text {coa }} .
$$

The single-layer equation (7) will be used in this form in the numerical examples of Section 8. In terms of the new discrete density $\hat{\boldsymbol{\mu}}_{\text {соа }}=\mathbf{R} \tilde{\boldsymbol{\mu}}_{\text {coa }}$ one can also write (18) in left preconditioned form

$$
\left(\mathbf{I}_{\mathrm{coa}}+\mathbf{R K}_{\mathrm{coa}}^{\circ}\right) \hat{\boldsymbol{\mu}}_{\mathrm{coa}}=\mathbf{R g}_{\mathrm{coa}} .
$$

The double-layer equation (9) will be used in this form in the more elaborate scheme for complicated unit cells developed in Section 7 .

Functionals on $\mu(z)$ of the type

$$
\int f(z) \mu(z) \mathrm{d} z=\int f(z(t)) \mu(z(t)) z^{\prime}(t) \mathrm{d} t
$$

where $f(z)$ is a piecewise smooth function, assume the discretized form

$$
\mathbf{f}_{\text {coa }}^{T} \mathbf{Z}_{\text {соа }} \hat{\boldsymbol{\mu}}_{\mathrm{coa}},
$$

where $\mathbf{f}$ is a column vector and $\mathbf{Z}$ is a matrix containing discrete values $z_{i}^{\prime}=z^{\prime}\left(t_{i}\right)$ multiplied with weights $w_{i}$ on the diagonal. 

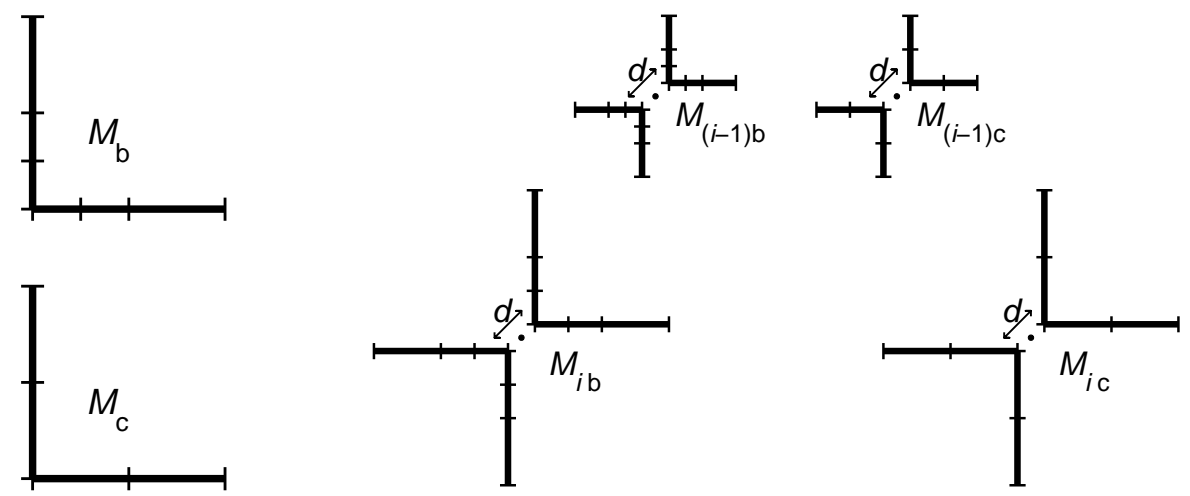

Figure 4: Local meshes close to corner vertices. Left: the square array. Right: the staggered array with meshes centered around a double-corner concentration point $\delta_{k}$. The vertex separation distance is $d$. Meshes with index $i=n_{\text {rec }}$ have the largest panels and $\mathcal{M}_{n c}$ coincides with the coarse mesh on $\Gamma_{0}$ in a neighborhood of $\delta_{k}$, see the rightmost image of Fig. 1 . The panels on meshes with index $i-1$ are half the size of those on meshes with index $i$.

\section{Recursive construction of $\mathbf{R}$}

The compressed inverse $\mathbf{R}$ has the same block diagonal structure as $\mathbf{K}_{\text {coa }}^{\star}$, see Section 4. Its construction from the definition (15) is costly when the refined mesh has many panels. Actually, the number of subdivisions needed to reach a given accuracy may grow without bounds due to the singularities in $\mu(z)$ that arise as $\sigma_{2} / \sigma_{1}$ approaches certain values, see Section 3 ,

Fortunately, the construction of each block $\mathbf{R}_{k}$ of $\mathbf{R}$, associated with a corner vertex $\gamma_{k}$ or with a double-corner concentration point $\delta_{k}$, can be greatly sped up and also stabilized via a recursion. This recursion uses grids on local meshes centered around a $\gamma_{k}$ or a $\delta_{k}$, see Figs. 3 and 4 .

\subsection{General recursion}

The staggered array of squares needs the recursion in the general form

$$
\mathbf{R}_{i k}=\mathbf{P}_{W \mathrm{bc}}^{T}\left(\mathbb{F}\left\{\mathbf{R}_{(i-1) k}^{-1}\right\}+\mathbf{I}_{\mathrm{b}}^{\circ}+\mathbf{K}_{i \mathrm{~b} k}^{\circ}\right)^{-1} \mathbf{P}_{\mathrm{bc}}, \quad i=1, \ldots, n_{\mathrm{rec}},
$$

where the number of recursion steps $n_{\text {rec }}$ corresponds to $n_{\text {sub }}$ of the refined mesh and where $\mathbf{R}_{i k}=\mathbf{R}_{k}$ for $i=n_{\text {rec }}$. See Section 6 of [13]. The weighted and unweighted prolongation operators $\mathbf{P}_{W \mathrm{bc}}$ and $\mathbf{P}_{\mathrm{bc}}$ act from a 128-point grid on a local mesh $\mathcal{M}_{i c}$ to a 192 -point grid on a local mesh $\mathcal{M}_{i \mathrm{~b}}$, see the 
right image of Fig. 4. The superscript 'o' in (22) has a meaning which can be explained by considering the discretization of $K$ on a 192-point grid on $\mathcal{M}_{i \mathrm{~b}}$ and on a 128-point grid on $\mathcal{M}_{(i-1) \mathrm{c}}$. Let the resulting matrices be $\mathbf{K}_{i \mathrm{~b} k}$ and $\mathbf{K}_{(i-1) \mathrm{ck}}$. Now $\mathbf{K}_{i \mathrm{~b} k}^{\circ}$ is the $192 \times 192$ matrix which results from zeroing all entries of $\mathbf{K}_{i \mathrm{~b} k}$ that also are contained in the $128 \times 128$ matrix $\mathbf{K}_{(i-1) \mathrm{c} k}$. The operator $\mathbb{F}\{\cdot\}$ expands an $128 \times 128$ matrix into an $192 \times 192$ matrix by zero-padding in such a way that $\mathbb{F}\left\{\mathbf{K}_{(i-1) \mathrm{c} k}\right\}+\mathbf{K}_{i \mathrm{~b} k}^{\circ}=\mathbf{K}_{i \mathrm{~b} k}$.

\subsection{Fixed-point iteration and Newton's method}

The recursion (22) can be simplified for square arrays of squares and for checkerboards thanks to scale invariance of the integrals in (17) and (9). The local meshes $\mathcal{M}_{i \mathrm{~b}}$ and $\mathcal{M}_{i \mathrm{c}}$ look the same at all recursion steps and the index $i$ can be dropped, see the right image of Fig. 3 and the left image of Fig. 4 . The recursion (22) assumes the form of a fixed-point iteration

$$
\mathbf{R}_{i k}=\mathbf{P}_{W \mathrm{bc}}^{T}\left(\mathbb{F}\left\{\mathbf{R}_{(i-1) k}^{-1}\right\}+\mathbf{I}_{\mathrm{b}}^{\circ}+\mathbf{K}_{\mathrm{b} k}^{\circ}\right)^{-1} \mathbf{P}_{\mathrm{bc}}, \quad i=1, \ldots, n_{\mathrm{rec}},
$$

which for $n_{\text {rec }} \rightarrow \infty$ can be cast as a non-linear matrix equation

$$
\mathbf{G}\left(\mathbf{R}_{k}\right) \equiv \mathbf{P}_{W \mathrm{bc}}^{T} \mathbf{A}\left(\mathbf{R}_{k}\right) \mathbf{P}_{\mathrm{bc}}-\mathbf{R}_{k}=0
$$

where

$$
\mathbf{A}\left(\mathbf{R}_{k}\right)=\left(\mathbb{F}\left\{\mathbf{R}_{k}^{-1}\right\}+\mathbf{I}_{\mathrm{b}}^{\circ}+\mathbf{K}_{\mathrm{b} k}^{\circ}\right)^{-1},
$$

see Sections 3.2 and 3.3 of [15]. The non-linear equation (24), in turn, can be solved for $\mathbf{R}_{k}$ with a variant of Newton's method. Let $\mathbf{X}$ be a matrix-valued perturbation of $\mathbf{R}_{k}$ and expand $\mathbf{G}\left(\mathbf{R}_{k}+\mathbf{X}\right)=0$ to first order in $\mathbf{X}$. This gives a Sylvester-type matrix equation

$$
\mathbf{X}-\mathbf{P}_{W \mathrm{bc}}^{T} \mathbf{A}\left(\mathbf{R}_{k}\right) \mathbb{F}\left\{\mathbf{R}_{k}^{-1} \mathbf{X} \mathbf{R}_{k}^{-1}\right\} \mathbf{A}\left(\mathbf{R}_{k}\right) \mathbf{P}_{\mathrm{bc}}=\mathbf{G}\left(\mathbf{R}_{k}\right)
$$

for the Newton update $\mathbf{X}$. One can use the Matlab built-in function dlyap for (26), but GMRES [28] gives a smaller residual and we use that method.

\subsection{Initialization, number of recursion steps, and homotopy}

The recursion (22), the fixed-point iteration (23), and Newton's method for (24) need to be initialized and $n_{\text {rec }}$ has to be decided in (22) and in (23). The three types of domains call for different strategies. 


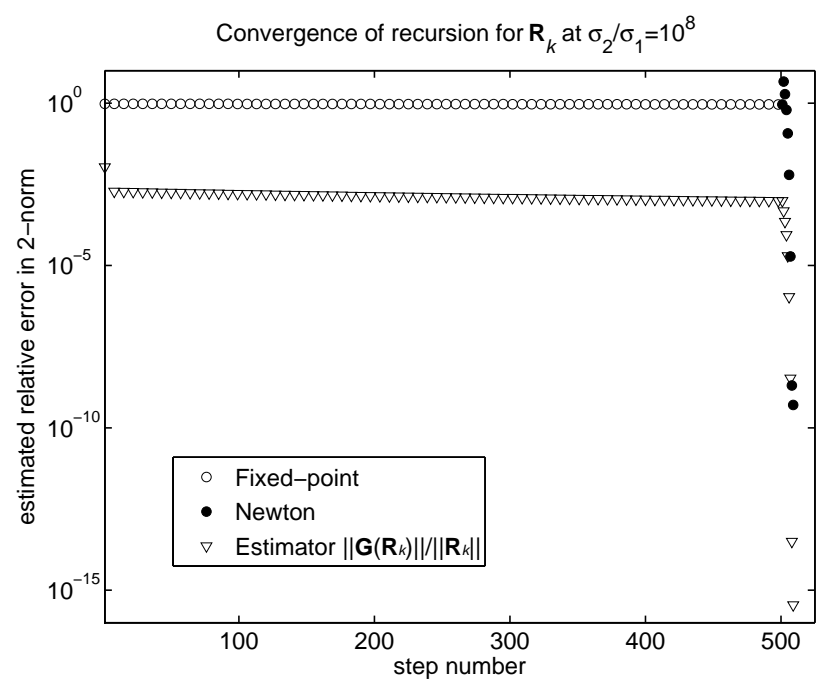

Figure 5: Convergence of $\mathbf{R}_{k}$ associated with a $\gamma_{k}$ of an ordered checkerboard with $\sigma_{2} / \sigma_{1}=$ $10^{8}$. The 500 fixed-point iteration steps in (23) are followed by 9 Newton steps for (24).

Random checkerboards at high contrast ratios are the easiest to deal with. Here we first use the fixed-point iteration (23), initialized with

$$
\mathbb{F}\left\{\mathbf{R}_{0 k}^{-1}\right\}=\mathbf{I}_{\mathrm{c}}+\mathbf{K}_{\mathrm{c} k},
$$

where subscript 'c' refers to a discretization on mesh $\mathcal{M}_{\mathrm{c}}$ in Fig. 3. Compare eq. (24) of [15]. The iterations are stopped when either $\left\|\mathbf{G}\left(\mathbf{R}_{k}\right)\right\| /\left\|\mathbf{R}_{k}\right\|$ is smaller than $10 \epsilon_{\text {mach }}$ in Frobenius norm or a number of 500 iterations is reached. The final fixed-point iterate is then used as initial guess in Newton's method for (24). The Newton iterations are stopped when either $\left\|\mathbf{G}\left(\mathbf{R}_{k}\right)\right\| /\left\|\mathbf{R}_{k}\right\|$ is smaller than $100 \epsilon_{\text {mach }}$ or a maximum number of 15 iterations is reached. Fig. 5 illustrates this strategy for an $\mathbf{R}_{k}$ associated with a $\gamma_{k}$ of an ordered checkerboard at $\sigma_{2} / \sigma_{1}=10^{8}$. One can see that the convergence of the fixed-point iteration is very slow. About $2 \cdot 10^{5}$ steps, corresponding to the same number of subdivisions of the fine mesh, would be needed for full convergence if only the fixed-point iteration was used. This clearly shows the power of Newton iterations and explains why methods relying solely on mesh refinement run into great difficulties on these types of domains. There are only 16 possible corner configurations in a random checkerboard, corresponding to 16 distinct blocks of $\mathbf{R}$. Therefore, the time- and storage requirements 
for computing $\mathbf{R}$ are negligible for large unit cells.

Square arrays of squares at negative contrast ratios are more difficult to treat. This is so since the solution to the electrostatic equation may only exist as a limit for $\sigma_{2} / \sigma_{1}$ approaching the negative real axis, see Section 2.1. Again we first use the fixed-point iteration (23), initialized as in (27) but with $\sigma_{2}$ (which enters into $\mathbf{K}_{\mathrm{b} k}^{\circ}$ ) multiplied with a constant $q=1-0.01 \mathrm{i}$. Again the final fixed-point iterate is used as initial guess in Newton's method for (24). Now, however, we use a homotopy method and at each Newton step we reduce the imaginary part of $q$ with a factor of ten. After 14 such iterations $q$ is set to unity and an additional maximum of 15 Newton iterations are performed. In this way the final expression for $\mathbf{R}_{k}$ may be complex valued even though the last few matrices $\mathbf{K}_{\mathrm{b} k}^{\circ}$, fed into (24), are purely real.

Staggered arrays of squares at negative contrast ratios are the most intricate. Here the choice of $n_{\text {rec }}$ and of initializer in (22) are very important. We choose $n_{\text {rec }}$ large enough so that the vertex separation distance $d$, see Fig. 4 , at the first recursion step $(i=1)$ is at least $10^{16}$ times larger than the part of $\Gamma_{k}^{\star}$ covered by the mesh $\mathcal{M}_{1 b}$. In this way the interaction between the two connected parts of $\mathcal{M}_{1 b}$ is negligible. The initializer $\mathbf{R}_{0 k}$ is then chosen as a compressed inverse computed using the homotopy method just described for the $\mathbf{R}_{k}$ of the square array of squares, neglecting the interaction between the connected parts of $\mathcal{M}_{1 b}$.

\section{Long-range preconditioning}

As the number of squares in a unit cell grows, the problem of computing $\sigma_{*}$ gets harder, see Section 1.1. This section improves on Section 4 of [15] and describes a long-range preconditioner for (9) which cures these problems. The main idea is to split the unknown layer density into two parts and capture all long-range interaction in a matrix $\mathbf{S}$, which can be rapidly inverted and used in a right-preconditioner. In combination with the short-range preconditioner $\mathbf{R}$ of Section 5, applied from the left, this results in a scheme whose computational cost for checkerboards with large random unit cells at high contrast ratios is almost linear in $N_{\text {sq }}$.

\subsection{An expanded equation}

Each square in $D_{0}$ has a boundary consisting of four straight segments, see Fig. 2. Introduce piecewise constant local basis functions $s_{k}(z), k=$ $1, \ldots, N_{\mathrm{sq}}$, on $\Gamma_{0} \cup L_{0}$ such that $s_{k}(z)=1$ when $z$ lies on a boundary part 
of square $k$ with positive orientation, $s_{k}(z)=-1$ when $z$ lies on a boundary part with negative orientation, and $s_{k}(z)=0$ otherwise.

Following Section 4.1 of [15], we take (9) in the general form (1) and expand it into the system

$$
\begin{gathered}
(I+K) \mu_{0}(z)+\sum_{k=1}^{N_{\mathrm{sq}}-1} a_{k}\left(s_{k}(z)-\lambda(z)\left|s_{k}(z)\right|+\frac{2 \lambda(z)}{N_{\mathrm{sq}}}\right)=g(z) . \\
\int s_{k}(z) \mu_{0}(z) \mathrm{d}|z|=0, \quad k=1, \ldots, N_{\mathrm{sq}}-1,
\end{gathered}
$$

where $\mu_{0}(z)$ mimics the rapidly varying behavior of $\mu(z)$ and $a_{k}$ are unknown coefficients.

Discretization of (28) and (29) together with left preconditioned compression, compare (19), results in the linear system

$$
\begin{gathered}
\left(\mathbf{I}_{\text {coa }}+\mathbf{R} \mathbf{K}_{\text {coa }}^{\circ}\right) \hat{\boldsymbol{\mu}}_{0 \mathrm{coa}}+\mathbf{R}\left(\mathbf{B}_{1}-\boldsymbol{\Lambda}_{\mathrm{coa}}\left|\mathbf{B}_{1}\right|+\boldsymbol{\lambda}_{\mathrm{coa}} \mathbf{u}^{T}\right) \mathbf{a}=\mathbf{R g}_{\mathrm{coa}}, \\
\mathbf{B}_{1}^{T}\left|\mathbf{Z}_{\mathrm{coa}}\right| \hat{\boldsymbol{\mu}}_{0 \mathrm{coa}}=\mathbf{0} .
\end{gathered}
$$

Here $\mathbf{B}_{1}$ is a $N_{\text {coa }} \times\left(N_{\mathrm{sq}}-1\right)$ matrix whose $k$ th column is the discretization of $s_{k}(z), \boldsymbol{\lambda}_{\text {coa }}$ is a column vector whose $N_{\text {coa }}$ entries is the discretization of $\lambda(z), \boldsymbol{\Lambda}_{\text {coa }}$ is matrix containing $\boldsymbol{\lambda}_{\text {coa }}$ on the diagonal, $\mathbf{u}$ is a column vector with $N_{\text {sq }}-1$ entries all equal to $2 / N_{\text {sq }}$, a is a column vector containing the $N_{\text {sq }}-1$ coefficients $a_{k}$, and vertical bars denote entrywise absolute value.

The effective conductivity (10) can be computed from

$$
\sigma_{*}=\Im\left\{\overline{\mathbf{e}}_{\mathrm{coa}}^{T} \mathbf{Z}_{\mathrm{coa}}\left(\hat{\boldsymbol{\mu}}_{0 \mathrm{coa}}+\mathbf{B}_{1} \mathbf{a}\right)\right\}
$$

once (30) and (31) is solved. Compare (21).

\subsection{An important simplification}

The definition of $s_{k}(z)$ together with Cauchy's integral theorem implies that

$$
\overline{\mathbf{e}}_{\mathrm{coa}}^{T} \mathbf{Z}_{\mathrm{coa}} \mathbf{B}_{1}=\mathbf{0} .
$$

As a consequence, the second term within parenthesis in (32) does not contribute to $\sigma_{*}$ and can be omitted. 
The fact that the coefficients $a_{k}$ are not needed in (32) opens up for another, more important, simplification. With the change of variables

$$
b_{k}=a_{k}+\frac{\left(\sigma(k)-\sigma\left(N_{\mathrm{sq}}\right)\right)}{\sigma\left(N_{\mathrm{sq}}\right)} \frac{\sum_{i=1}^{N_{\mathrm{sq}}-1} a_{i}}{N_{\mathrm{sq}}},
$$

where $\sigma(k)$ denotes the conductivity of square $k$, the expanded equation (28) assumes the simpler form

$$
(I+K) \mu_{0}(z)+\sum_{k=1}^{N_{\mathrm{sq}}-1} b_{k}\left(s_{k}(z)-\lambda(z)\left|s_{k}(z)\right|\right)=g(z)
$$

and (30) reduces to

$$
\left(\mathbf{I}_{\text {coa }}+\mathbf{R K}_{\text {coa }}^{\circ}\right) \hat{\boldsymbol{\mu}}_{0 \mathrm{coa}}+\mathbf{R}\left(\mathbf{B}_{1}-\boldsymbol{\Lambda}_{\text {coa }}\left|\mathbf{B}_{1}\right|\right) \mathbf{b}=\mathbf{R g}_{\text {coa }} .
$$

\subsection{A Schur complement style preconditioner}

The system (36) and (31) can be written in partitioned form

$$
\left[\begin{array}{cc}
\mathbf{I}+\mathbf{R K}^{\circ} & \mathbf{B} \\
\mathbf{C} & \mathbf{0}
\end{array}\right]\left[\begin{array}{c}
\hat{\boldsymbol{\mu}}_{0} \\
\mathbf{b}
\end{array}\right]=\left[\begin{array}{c}
\mathbf{R g} \\
0
\end{array}\right]
$$

where subscripts 'coa' are omitted and

$$
\begin{aligned}
& \mathbf{B}=\mathbf{R}\left(\mathbf{B}_{1}-\boldsymbol{\Lambda}_{\text {coa }}\left|\mathbf{B}_{1}\right|\right), \\
& \mathbf{C}=\mathbf{B}_{1}^{T}\left|\mathbf{Z}_{\text {coa }}\right| .
\end{aligned}
$$

The change of variables

$$
\left[\begin{array}{c}
\hat{\boldsymbol{\mu}}_{0} \\
\mathbf{b}
\end{array}\right]=\left[\begin{array}{cc}
\mathbf{I} & \mathbf{B} \\
\mathbf{C} & \mathbf{0}
\end{array}\right]^{-1}\left[\begin{array}{c}
\boldsymbol{\omega} \\
\mathbf{c}
\end{array}\right]=\left[\begin{array}{cc}
\mathbf{I}-\mathbf{B S}^{-1} \mathbf{C} & \mathbf{B S}^{-1} \\
\mathbf{S}^{-1} \mathbf{C} & -\mathbf{S}^{-1}
\end{array}\right]\left[\begin{array}{c}
\boldsymbol{\omega} \\
\mathbf{c}
\end{array}\right],
$$

where the $\left(N_{\mathrm{sq}}-1\right) \times\left(N_{\mathrm{sq}}-1\right)$ matrix $\mathbf{S}$ is given by

$$
\mathrm{S}=\mathrm{CB}
$$

transforms (37) into

$$
\left[\begin{array}{cc}
\mathbf{I}+\mathbf{R K}^{\circ}\left(\mathbf{I}-\mathbf{B S}^{-1} \mathbf{C}\right) & \mathbf{R K}^{\circ} \mathbf{B S}^{-1} \\
\mathbf{0} & \mathbf{I}
\end{array}\right]\left[\begin{array}{c}
\boldsymbol{\omega} \\
\mathbf{c}
\end{array}\right]=\left[\begin{array}{c}
\mathbf{R g} \\
0
\end{array}\right] .
$$

From (42) it is obvious that $\mathbf{c}=\mathbf{0}$ and we can write (42) as a single equation for $\boldsymbol{\omega}$ :

$$
\left(\mathbf{I}+\mathbf{R K}^{\circ}\left(\mathbf{I}-\mathbf{B S}^{-1} \mathbf{C}\right)\right) \boldsymbol{\omega}=\mathbf{R g} .
$$

The effective conductivity (32) can be expressed in terms of $\boldsymbol{\omega}$ as

$$
\sigma_{*}=\Im\left\{\overline{\mathbf{e}}_{\mathrm{coa}}^{T} \mathbf{Z}_{\mathrm{coa}}\left(\mathbf{I}-\mathbf{B S}^{-1} \mathbf{C}\right) \boldsymbol{\omega}\right\} .
$$




\subsection{The inverse of $\mathbf{S}$}

The matrix $\mathbf{S}$ of (41) is sparse. In the limit $\lambda(z) \rightarrow 0$ it approaches a standard five-point stencil for the discrete Laplace operator. As we soon shall see, MATLAB's sparse banded solver, obtained using 'backslash', is very efficient at solving linear systems with $\mathbf{S}$ as system matrix, at least for system sizes up to $N_{\mathrm{sq}}=1.6 \cdot 10^{6}$. We shall use that method in all numerical examples.

The condition number of $\mathbf{S}$ seems to be lower when $\sigma\left(N_{\mathrm{sq}}\right)=\sigma_{2}$ than when $\sigma\left(N_{\mathrm{sq}}\right)=\sigma_{1}$. Therefore, in our numerical examples, we permute the unit cell so that $\sigma\left(N_{\mathrm{sq}}\right)=\sigma_{2}$.

\subsection{Reduction in the number of unknowns}

Some entries of $\boldsymbol{\omega}$ in (43) are easy to solve for. To see this, let $\Gamma_{\text {eq }}$ be the part of $\Gamma_{0}$ that lies between squares of equal conductivity. From (6) it follows that $\lambda(z)=0$ for $z \in \Gamma_{\text {eq }}$. This means that all entries of $\mathbf{K}^{\circ}$ and $\mathbf{R}$ whose first index corresponds to a discretization point $z_{i} \in \Gamma_{\text {eq }}$ are zero except for the diagonal entries $R_{i i}$ which are one. From (43) we get the simple entrywise relation

$$
\omega_{i}=g_{i}, \quad z_{i} \in \Gamma_{\mathrm{eq}} .
$$

Furthermore, the vast majority of these elements $g_{i}$ are zero thanks to $l_{0}(z)$, see the right hand side of (91).

Eq. (45) can be used to reduce the number of unknowns in (43). The savings are huge when the area fraction $p$ is high or low. For simplicity, we only remove the known entries of $\boldsymbol{\omega}$ which are zero. The reduced system assumes the form

$$
\left(\downarrow \overrightarrow{\mathbf{I}}+\downarrow \overrightarrow{\mathbf{R}} \downarrow \mathbf{K}^{\circ}\left(\overrightarrow{\mathbf{I}}-\mathbf{B S}^{-1} \overrightarrow{\mathbf{C}}\right)\right) \boldsymbol{\omega}_{\mathrm{u}}=\downarrow \mathbf{R g} .
$$

Here $\boldsymbol{\omega}_{u}$ are the remaining entries of $\boldsymbol{\omega}$, 'downarrow' indicates that rows of a matrix are deleted, and 'rightarrow' indicates that columns are deleted. One can see in (46) that the reduction in the number of unknowns does not induce a similar reduction in the size of $\mathbf{K}^{\circ}$. No columns are deleted. Therefore, the speedup resulting from (46) is not as great as the savings in storage.

\section{Numerical examples}

This section investigates the complexity and the achievable accuracy of our scheme (18) for ordered arrays of squares and (43) and (46) for random 
checkerboards. We also compare with recent numerical results [6] obtained with the finite element solver ABACUS.

The numerical examples are performed in the MATLAB environment (version 7.9). The GMRES iterative solver [28] and a threaded version of the fast multipole method [11], coded in C with SIMD instructions, is used for the main linear systems. The stopping criterion threshold is set to machine epsilon $\left(\epsilon_{\text {mach }}\right)$. See Section 4.1 of [11] and Section 3 of [9] for how to impose periodic boundary conditions on potential fields due to charges in a unit cell. The examples involving $N_{\text {sq }}$ up to around $10^{6}$ are executed on a workstation equipped with an IntelXeon E5430 CPU at $2.66 \mathrm{GHz}$ and $32 \mathrm{~GB}$ of memory while all other examples are executed on a workstation equipped with an IntelCore2 Duo E8400 CPU at $3.00 \mathrm{GHz}$ and $4 \mathrm{~GB}$ of memory.

When estimating accuracy we rely on some exact relations available for two-component media and compiled in Chapters 3.2 and 8.7 of Milton [25]. Let us consider $\sigma_{*}$ and the effective conductivity tensor $\boldsymbol{\sigma}_{*}$ as functions of $\sigma_{1}$ and $\sigma_{2}$. Then, using a duality transform and the homogeneity of $\boldsymbol{\sigma}_{*}$, one can show the following relation between an original material and that of a material where the components have been interchanged

$$
\boldsymbol{\sigma}_{*}\left(\sigma_{2}, \sigma_{1}\right)=\sigma_{1} \sigma_{2} \boldsymbol{\sigma}_{*}\left(\sigma_{1}, \sigma_{2}\right) / \operatorname{det}\left(\boldsymbol{\sigma}_{*}\left(\sigma_{1}, \sigma_{2}\right)\right) .
$$

Another useful relation which holds for overall isotropic materials is

$$
\sigma_{*}\left(\sigma_{1}, \sigma_{2}\right) \bar{\sigma}_{*}\left(1 / \bar{\sigma}_{1}, 1 / \bar{\sigma}_{2}\right)=1
$$

An ordered checkerboard has

$$
\sigma_{*}=\sqrt{\sigma_{1} \sigma_{2}}
$$

and a square array of squares at $p=0.25$ has

$$
\sigma_{*}=\sigma_{1} \sqrt{\left(\sigma_{1}+3 \sigma_{2}\right) /\left(3 \sigma_{1}+\sigma_{2}\right)} .
$$

We also observe, see Chapter 1.7 of [25], that the effective conductivity of a random checkerboard at $p=0.5$ obeys

$$
\lim _{N_{\mathrm{sq}} \rightarrow \infty} \sigma_{*}=\sqrt{\sigma_{1} \sigma_{2}} .
$$

Note that the tensor $\boldsymbol{\sigma}_{*}$ has four elements and that they all can be computed via (43) (or (46)) and (44). For example, choosing $e=1$ both in (43), where $e$ appears in $\mathbf{g}$, and in (44) makes $\sigma_{*}$ assume the value of $\sigma_{* x x}$. Choosing $e=1$ in (43) and $e=\mathrm{i}$ in (44) makes $\sigma_{*}$ assume the value of $\sigma_{* y x}$. 

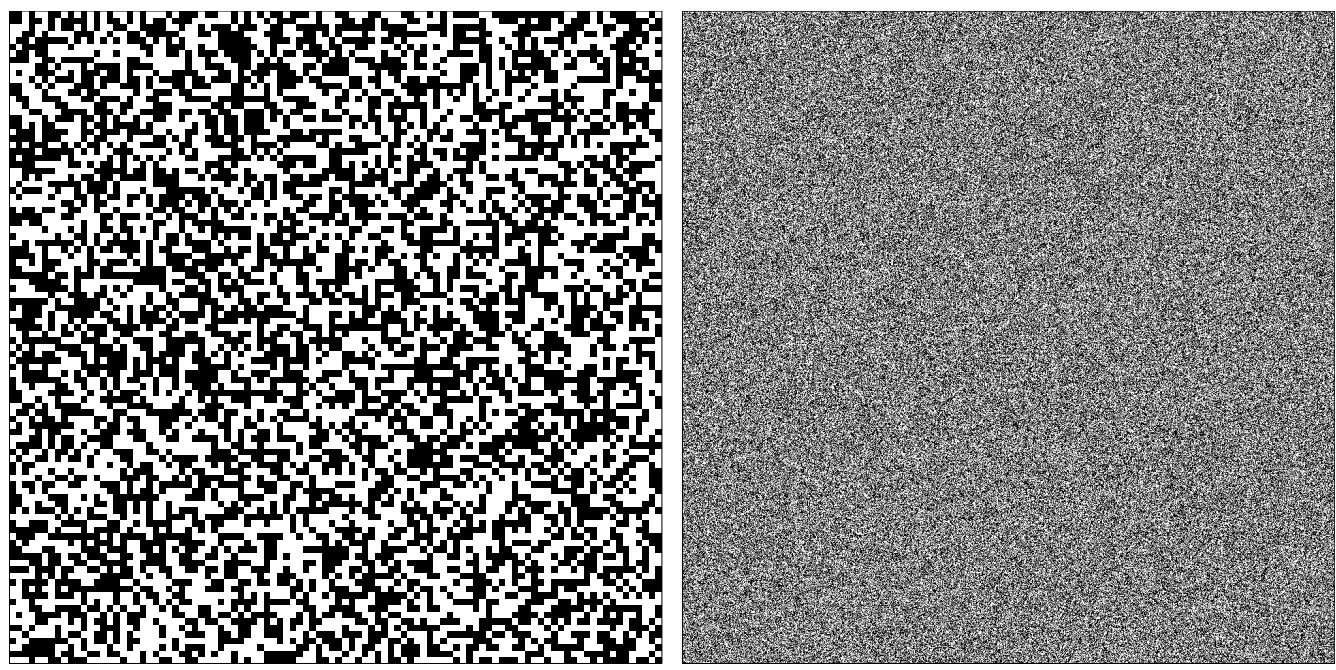

Figure 6: Unit cells of two random checkerboards used in the numerical examples. The area fraction of squares with conductivity is $\sigma_{2}$ is $p=0.5$. Left: $N_{\mathrm{sq}}=10^{4}$. Right: $N_{\mathrm{sq}}=10^{6}$. Please zoom to see the details of the microstructure in the right image.

\subsection{Timing and convergence to statistical limit}

A sequence of 105 random checkerboards is constructed with unit cell sizes ranging from $N_{\mathrm{sq}}=4$ to $N_{\mathrm{sq}}=1615441$. All unit cells have $\sigma_{2} / \sigma_{1}=10^{6}$ and $p=0.5$, see Fig. 6 for two layouts. The effective conductivities $\sigma_{* y y}$ of the checkerboards are computed via (46) and (44).

Almost all computing time is spent in the GMRES solver. The setup time for $\mathbf{S}$ of (41) at $N_{\mathrm{sq}}=10^{6}$, for example, is only about $0.4 \%$ of the total computing time. The number of iterations needed for full convergence is bounded by 18 and the left image of Fig. 7 shows that the time spent in GMRES grows approximately linearly with $N_{\text {sq }}$, reflecting the complexity of the fast multipole method. The total time spent applying the inverse of $\mathbf{S}$, which is included in the time spent in GMRES, is also shown separately in the left image of Fig. 7. One can see that while this time grows faster than linearly, it is still less than $9 \%$ of the total computing time at $N_{\mathrm{sq}}=10^{6}$.

The right image of Fig. 7 shows the actual values for the effective conductivities $\sigma_{*}$ of the checkerboards, presented in terms of their relative deviation from the statistical limit (51). At $N_{\mathrm{sq}}=1615441$, which is the largest unit cell we can handle due to memory constraints, the deviation is about $1 \%$. 

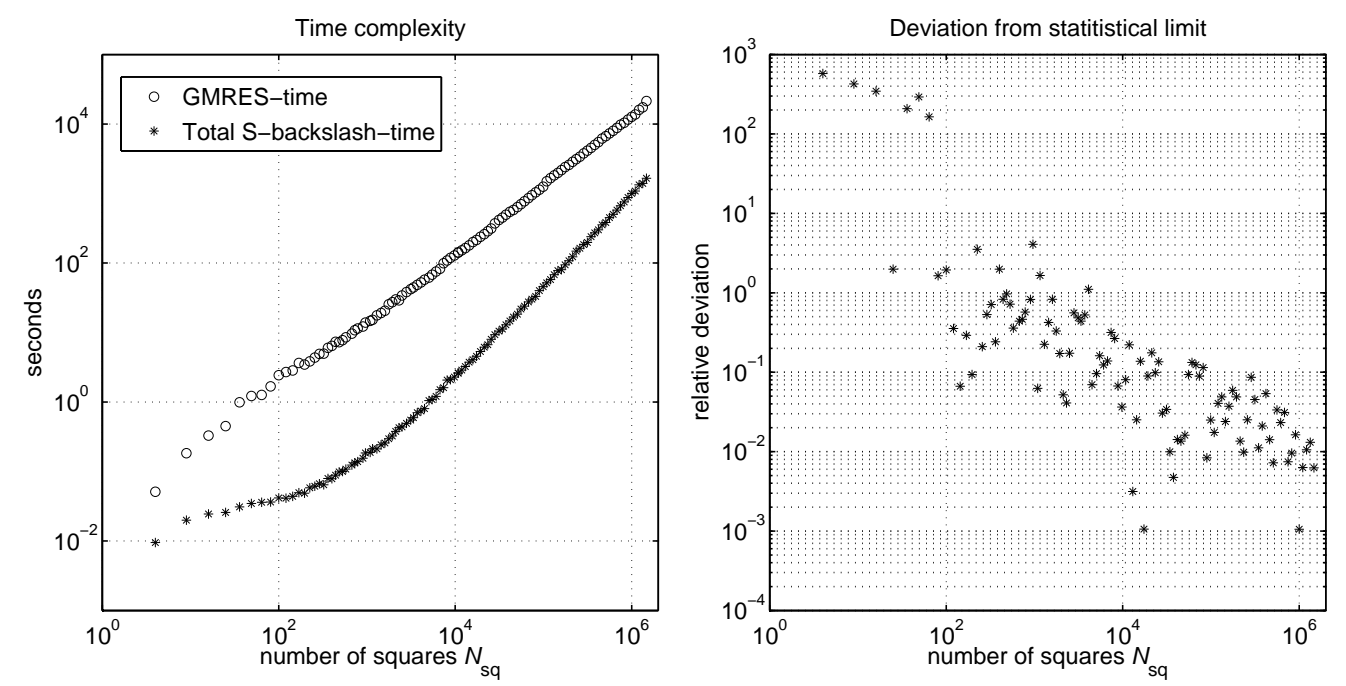

Figure 7: Solving for the effective conductivity of random checkerboards with $\sigma_{2} / \sigma_{1}=10^{6}$ and unit cells of increasing sizes. The area fraction of squares with conductivity $\sigma_{2}$ is $p=0.5$.

\subsection{Achievable accuracy}

Fig. 8, left image, illustrates how the placement of quadrature nodes influences the achievable accuracy for progressively higher contrast ratios. The unit cell is that of an ordered checkerboard with $N_{\mathrm{sq}}=4$. The circles show that the relative error in $\sigma_{*}$ grows roughly as $\left(\sigma_{2} / \sigma_{1}\right)^{1.5}$ when Legendre nodes are used on all panels. This was the strategy in [15]. The stars show that the growth rate becomes linear in $\sigma_{2} / \sigma_{1}$ when Jacobi nodes are used on special panels. This is the strategy of the present paper, see Section 3 . Several extra digits can be obtained at high contrast ratios.

Note that for $\sigma_{2} / \sigma_{1}>10^{16}$, accurate results are impossible in double precision arithmetic. This is so since $\lambda(z)$ of (7) and (9) is then indistinguishable from unity. The integral equations become independent of $\sigma_{2}$ while the reference solution (49) is not. The error growth rate produced by the Jacobi nodes in Fig. 8 could therefore be thought of as optimal.

Three sequences of checkerboards are now constructed with unit cell sizes ranging from $N_{\mathrm{sq}}=4$ to $N_{\mathrm{sq}}=1468944$ and with $p=0.5$. The first sequence consists of random checkerboards with $\sigma_{2} / \sigma_{1}=10^{6}$. The relative errors in 

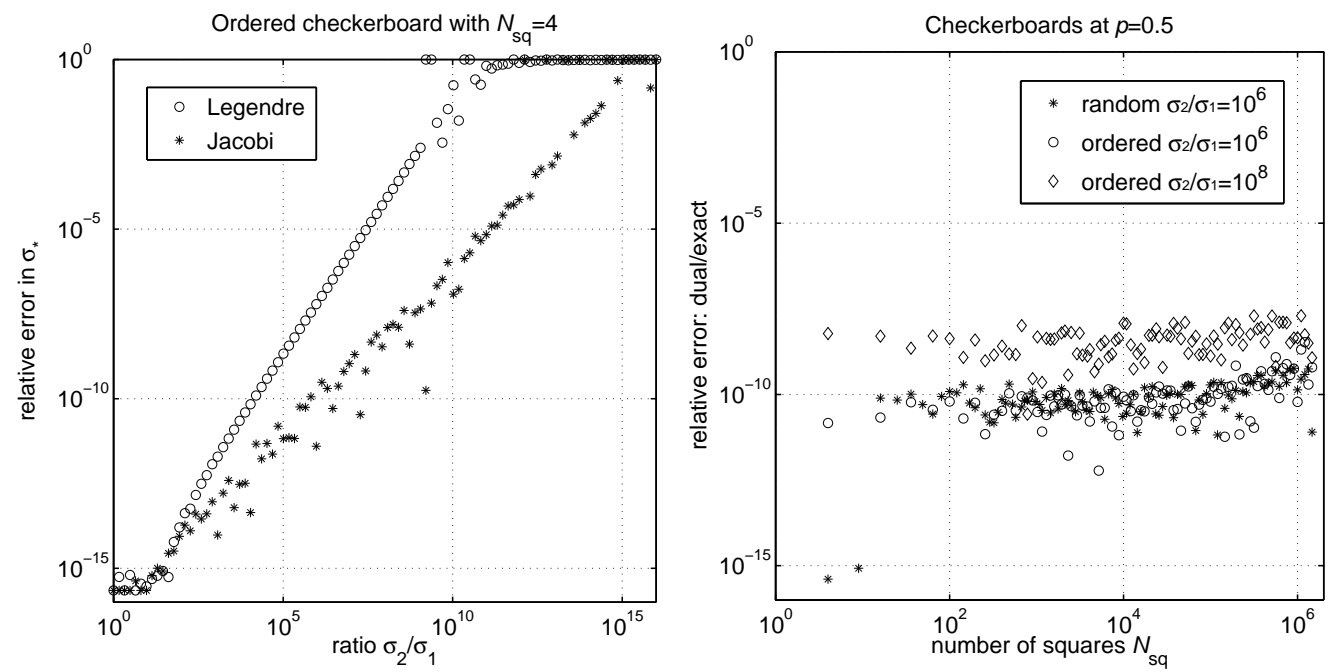

Figure 8: Achievable accuracy in the effective conductivity $\sigma_{*}$. Left: Two strategies for placing nodes on special panels are compared. Errors outside of the range $\left[\epsilon_{\mathrm{mach}}, 1\right]$ are shown as either $\epsilon_{\text {mach }}$ or 1 , whichever is closest. Right: error growth as a function of unit cell size.

their computed effective conductivities are estimated via (47) as

$$
\frac{\left\|\boldsymbol{\sigma}_{*}\left(\sigma_{2}, \sigma_{1}\right)-\sigma_{1} \sigma_{2} \boldsymbol{\sigma}_{*}\left(\sigma_{1}, \sigma_{2}\right) / \operatorname{det}\left(\boldsymbol{\sigma}_{*}\left(\sigma_{1}, \sigma_{2}\right)\right)\right\|_{2}}{\left\|\boldsymbol{\sigma}_{*}\left(\sigma_{2}, \sigma_{1}\right)\right\|_{2}} .
$$

The second sequence consists of ordered checkerboards with $\sigma_{2} / \sigma_{1}=10^{6}$ and (49) is used as reference solution. The third sequence is the same as the second sequence, but the contrast ratio is increased to $\sigma_{2} / \sigma_{1}=10^{8}$.

Fig. 8, right image, shows the results and it has several interesting features. For example, one can see that:

- the error in $\sigma_{*}$ seems to be independent of the unit cell size. This is so because the total error is dominated by the error caused by corner selfinteraction, computed in local coordinates. The error from long-range interaction is comparatively small except for $N_{\mathrm{sq}}>5 \cdot 10^{5}$.

- the error estimate for $\boldsymbol{\sigma}_{*}$ of random checkerboards (52), based on duality, agrees well with the error estimate for $\sigma_{*}$ of ordered checkerboards, based on an exact answer (49). 

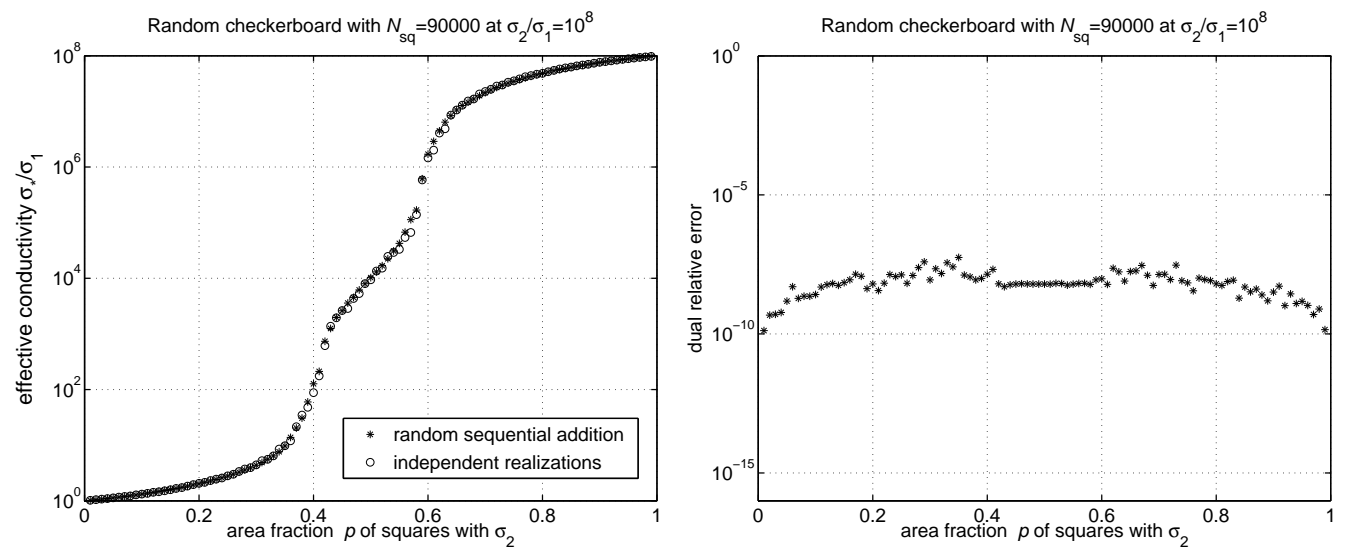

Figure 9: Random checkerboards with $N_{\mathrm{sq}}=90000$ squares in the unit cell and $\sigma_{2} / \sigma_{1}=10^{8}$. The area fraction of squares with conductivity $\sigma_{2}$ varies from $p=0$ to $p=1$.

\subsection{Continuum percolation}

In theoretical materials science it is of interest to study the effective conductivities of continuum two-component random composites as $p$ varies. Fig. 9 shows such a study for a unit cell with $N_{\mathrm{sq}}=90000$ and $\sigma_{2} / \sigma_{1}=10^{8}$ along with the error estimate (52). Two sequences of realizations are shown - one based on sequential random addition and one where all realizations are independent. It is obvious, from the jagged shape of the curve in the left image and also from the results in Section 8.1, that we are far from the statistical limit. Percolation thresholds are visible at $p \approx 0.41$ and at $p \approx 0.59$. These numbers are consistent with classic results on site percolation for a square lattice [22]. The overall behavior of $\sigma_{*}$ as a function of $p$ in Fig. 9 is in agreement with the discussion on p. 207 in Milton [25] and also with results obtained with a discrete network model [23] but it stands in contrast to results obtained with the finite element method in Fig. 2(a) of [6]. There only one percolation threshold is observed.

\subsection{The square array of squares}

Fig. 10] shows computed values of $\sigma_{*} / \sigma_{1}$ for the square array of squares at $p=0.25$ for negative ratios $\sigma_{2} / \sigma_{1}$. The relative error, with (50) as reference solution, is shown in the right image. The error is close to $\epsilon_{\text {mach }}$ except for in a neighborhood of three points where it is higher: the 'pole' or 'resonance' at $\sigma_{2} / \sigma_{1}=-3$, the 'essential singularity' at $\sigma_{2} / \sigma_{1}=-1$, and the 'zero' at 

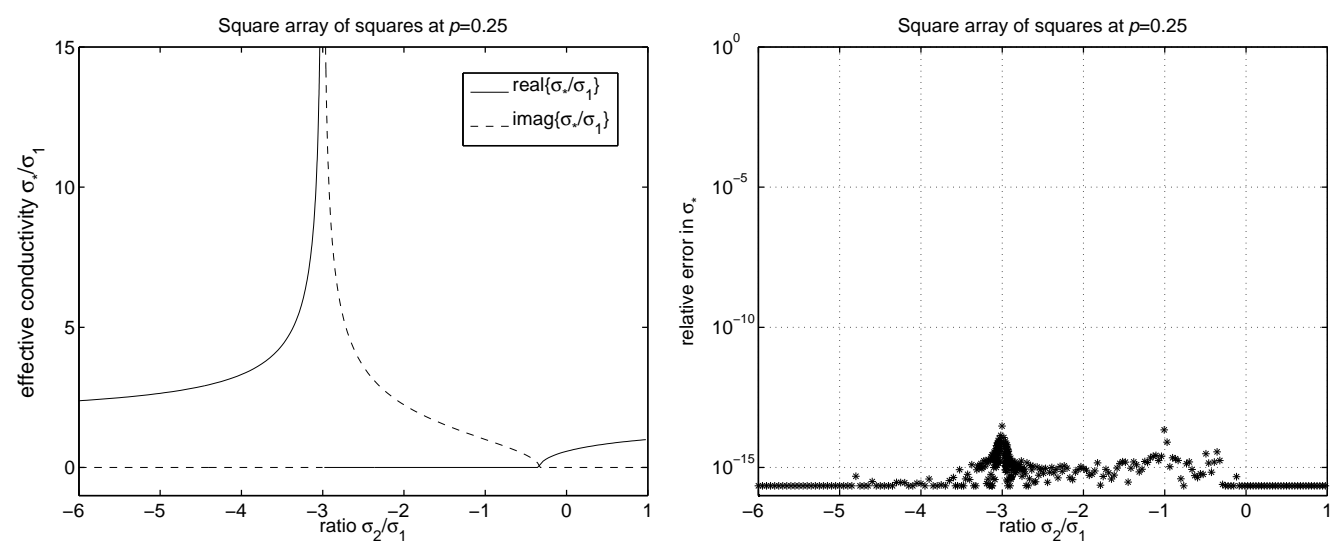

Figure 10: Left: the effective conductivity $\sigma_{*} / \sigma_{1}$ of a square array of squares at $p=0.25$. The curves are supported by 349 adaptively spaced data points. Right: the relative error with (50) as reference solution.

$\sigma_{2} / \sigma_{1}=-1 / 3$. See [27] for an explanation of the significance and physical meaning of these terms. Note that at $\sigma_{2} / \sigma_{1}=-1$ we have $\lambda= \pm \infty$ and that (77) then becomes a first kind equation. Compare also Fig. 2 of [27], which is similar to the left image of our Fig. 10, but where some problems are encountered along the branch cut $\sigma_{2} / \sigma_{1} \in[-3,-1 / 3]$.

\subsection{The staggered array of squares}

Staggered arrays of squares at area fractions close to $p=0.5$ exhibit rich resonant spectra on the negative real axis and pose greater challenges to numerics than the example of Section 8.4. More data points are required to resolve $\sigma_{*}$. For modeling purposes it is convenient to describe staggered arrays in terms of a parameter $d_{0}$, related to the area fraction $p$ and to the vertex separation distance $d$, see Figs. 1 and [4, as

$$
p=\frac{2}{\left(d_{0}+2\right)^{2}} \quad \text { and } \quad d=\frac{d_{0}}{\sqrt{2}\left(d_{0}+2\right)} .
$$

The left image of Fig. 11 for $d_{0}=10^{-10}$ shows an oscillatory behavior of $\sigma_{*} / \sigma_{1}$ for $\sigma_{2} / \sigma_{1} \in[-3,-1 / 3]$ and a number of resonances on the negative real axis outside of this interval. The right image shows that the relative error in these computations, estimated via how well (48) is met, is typically on the order of $10^{2} \epsilon_{\text {mach }}$. Close to the eigenvalues of (7), some of which 

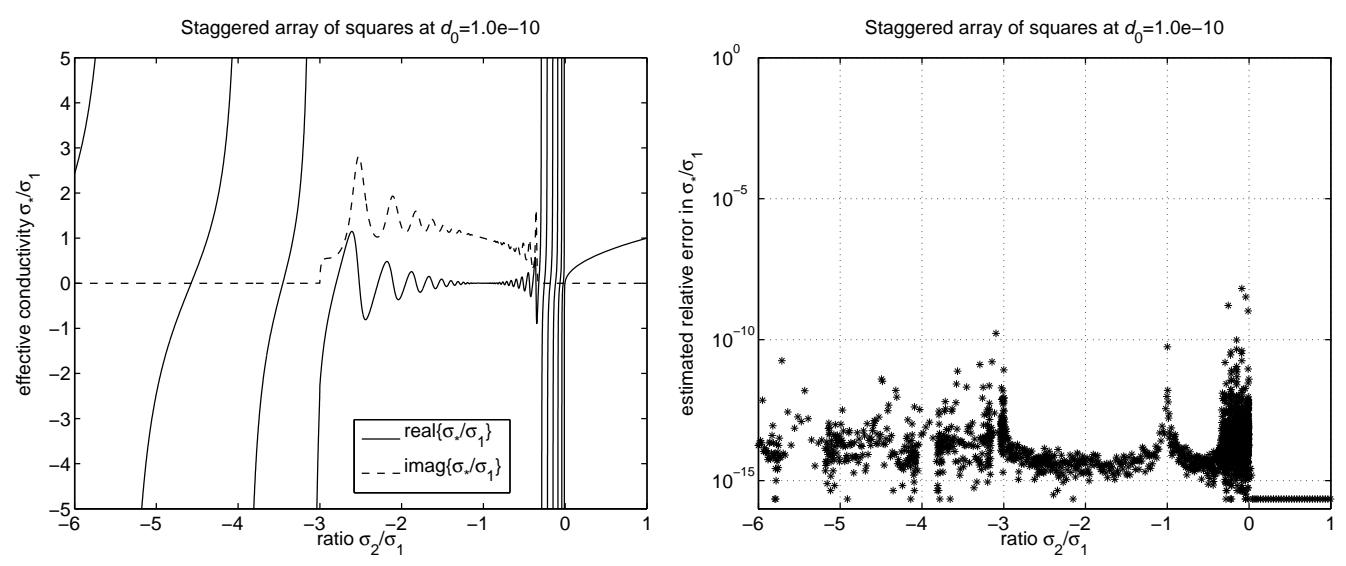

Figure 11: The effective conductivity $\sigma_{*} / \sigma_{1}$ of a staggered array of squares at $d_{0}=10^{-10}$. The curves are supported by 2006 data points. Right: an error estimate based on (48).

correspond to poles of $\sigma_{*}$, the error is of course larger. The largest relative error encountered in this example is estimated to $10^{-8}$.

\section{Conclusions}

The homogenization of composite materials with large random unit cells of squares at extreme material property ratios is a canonical problem in the theory of composite materials. It has fascinated researchers for decades [25]. The domains look simple, yet they are intriguing. There are analytical results available for special cases, yet numerical solvers run into trouble. Only a few years ago, numerical solutions to the type of homogenization problems presented in this paper would be considered far out of reach.

The present work epitomizes and stretches a recent line of research 13 , 14, 15, 17, 18] to a new high. We first show how to treat simple unit cells with (almost) optimal accuracy using a short-range preconditioner. We then show that larger unit cells pose no extra problems when a new long-range preconditioner is added. Our algorithm has (almost) linear complexity in both execution time and storage requirement. Problems involving unit cells with a million of squares can be solved to very high precision in a few hours. Homogenization on checkerboard-like domains have become a simple task.

How useful is our new scheme? The coupling of checkerboard problems to real-world physics is elusive. One may question the relevance of the small 
length-scales needed for the resolution of various singular fields. Nevertheless, a recent surge in physicists' interest in metamaterials has given new momentum to the study of these issues [27]. The difficulties arising in random checkerboard problems may, further, be representative of the sort of troubles that arise in several real-world problems. Since integral equation methods are widely applicable, it is therefore likely that our scheme and generalizations thereof have many immediate applications. Further work based on the present scheme and directed towards metamaterial applications is in progress [16].

\section{References}

[1] S.A. Berggren, D. Lukkassen, A. Meidell, L. Simula, A new method for numerical solution of checkerboard fields, J. Appl. Math., 1 (2001) $157-173$.

[2] J. Bremer Z. Gimbutas, and V. Rokhlin, A nonlinear optimization procedure for generalized Gaussian quadratures, SIAM J. Sci. Comput., 32 (2010) 1761-1788.

[3] J. Bremer and V. Rokhlin, Efficient discretization of Laplace boundary integral equations on polygonal domains, J. Comput. Phys., 229 (2010) $2507-2525$.

[4] J. Bremer, V. Rokhlin, and I. Sammis, Universal quadratures for boundary integral equations on two-dimensional domains with corners, J. Comput. Phys., 229 (2010) 8259-8280.

[5] O.P. Bruno, J.S. Oval, and C. Turc, A high-order integral algorithm for highly singular PDE solutions in Lipschitz domains, Computing, 84 (2009) 149-181.

[6] Y. Chen and C.A. Schuh, Effective transport properties of random composites: Continuum calculations versus mapping to a network, Phys. Rev. E, 80 (2009) 040103.

[7] D.R. Fredkin and I.D. Mayergoyz, Resonant Behaviour of Dielectric Objects (Electrostatic Resonances), Phys. Rev. Lett. 91 (2003) 253902. 
[8] A. Gillman, P. Young, and P.G. Martinsson, Numerical Homogenization via Approximation of the Solution Operator. In B. Engquist, O. Runborg, R. Tsai (Eds.), Numerical Analysis of Multiscale Computations, vol. 82 of Lect. Notes Comput. Sci. Eng., Springer, Heidelberg, 2011.

[9] L. Greengard and M.C. Kropinski, Integral equation methods for Stokes flow in doubly-periodic domains, J. Eng. Math. 48 (2004) 157-170.

[10] L. Greengard and J.-Y. Lee, Electrostatics and heat conduction in high contrast composite materials, J. Comput. Phys., 211 (2006) 64-76.

[11] L. Greengard and V. Rokhlin, A fast algorithm for particle simulations, J. Comput. Phys., 73 (1987) 325-348.

[12] R.F. Hamou, J.R. Macdonald, and E. Tuncer, Dispersive dielectric and conductive effects in 2D resistor-capacitor networks, J. Phys. Condens. Mat., 21 (2009) 025904.

[13] J. Helsing, Integral equation methods for elliptic problems with boundary conditions of mixed type, J. Comput. Phys., 228 (2009) 8892-8907.

[14] J. Helsing, A fast and stable solver for singular integral equations on piecewise smooth curves, SIAM J. Sci. Comput., 33 (2011) 153-174.

[15] J. Helsing, The effective conductivity of random checkerboards, J. Comput. Phys., 230 (2011) 1171-1181.

[16] J. Helsing, R.C. McPhedran, and G.W. Milton, Spectral superresolution in metamaterial composites, arXiv:1105.5012 v1 (submitted to New Journal of Physics).

[17] J. Helsing and R. Ojala, Corner singularities for elliptic problems: Integral equations, graded meshes, quadrature, and compressed inverse preconditioning, J. Comput. Phys., 227 (2008) 8820-8840.

[18] J. Helsing and R. Ojala, Elastostatic computations on aggregates of grains with sharp interfaces, corners, and triple-junctions, Int. J. Solids Struct., 46 (2009) 4437-4450.

[19] J.H. Hetherington and M.F. Thorpe, The conductivity of a sheet containing inclusions with sharp corners, Proc. R. Soc. Lond. A, 438 (1992) 591-604. 
[20] M.R. Karim and K. Krabbenhoft, New Renormalization Schemes for Conductivity Upscaling in Heterogeneous Media, Transp. Porous Med., 85 (2010) 677-690.

[21] I.C. Kim, An Efficient Brownian Motion Simulation Method for the Conductivity of a Digitized Composite Medium, KSME Internat. J., 17 (2003) 545-561.

[22] M.J. Lee, Pseudo-random-number generators and the square site percolation threshold, Phys. Rev. E, 78 (2008) 031131.

[23] E. Mårtensson and U. Gäfvert, Three-dimensional impedance networks for modelling frequency dependent electrical properties of composite materials, J. Phys. D, 36 (2003) 1864-1872.

[24] S.G. Mikhlin, Integral Equations and their applications to certain problems in mechanics, mathematical physics and technology, 2nd ed., Pergamon Press, London, 1964.

[25] G.W. Milton, The theory of composites, Cambridge University press, Cambridge, 2002.

[26] P.K.V.V. Nukala, S. Simunovic, S. Zapperi, and M.J. Alava, Fracture in three-dimensional random fuse model: recent advances through highperformance computing, J. Comput.-Aided Mater., 14 (2007) 25-35.

[27] W.T. Perrins and R.C. McPhedran, Metamaterials and the homogenization of composite materials, Metamaterials 4 (2010) 24-31.

[28] Y. Saad and M.H. Schultz, GMRES: A generalized minimal residual algorithm for solving nonsymmetric linear systems, SIAM J. Sci. Stat. Comp., 7 (1986) 856-869. 